\title{
A term neonate with early myoclonic encephalopathy caused by RARS2 gene variants: a case report
}

\author{
Yan $\mathrm{Xu}^{1 \#}$, Bing-Bing Wu ${ }^{2 \#}$, Hui-Jun Wang ${ }^{2}$, Shui-Zhen Zhou ${ }^{1}$, Guo-Qiang Cheng ${ }^{3}$, Yuan-Feng Zhou ${ }^{1}$ \\ ${ }^{1}$ Department of Neurology, ${ }^{2}$ The Molecular Genetic Diagnosis Center, Shanghai Key Lab of Birth Defects, Pediatrics Research Institute, \\ ${ }^{3}$ Department of Neonatology, Children's Hospital of Fudan University, Shanghai, China \\ \#These authors contributed equally to this work. \\ Correspondence to: Yuan-Feng Zhou; Guo-Qiang Cheng. Department of Neurology, Children's Hospital of Fudan University, No. 399 Wanyuan road, \\ Minhang District, Shanghai 201102, China. Email: yuanfengzhou99@163.com; gqchengcm@163.com.
}

\begin{abstract}
The RARS2 gene encodes mitochondrial arginine-tRNA synthetase. Patients with variants of the RARS2 gene have pontocerebellar hypoplasia type 6 (PCH6), which is characterized by early onset seizures, progressive microcephaly, and developmental delay. PCH6 is a rare mitochondrial encephalopathy. To the best of our knowledge, the onset seizure type which the ictal video-electroencephalogram (VEEG) was compatible with early myoclonic encephalopathy (EME) has not been reported. Here we reported a term female neonate with EME caused by heterozygous variants of the RARS2 gene [NM_020320: exon10: c.773G>A (p. R258H) Maternal, NM_020320: exon4: c.282_285delAGAG Paternal]. Groan was the first symptom manifested, followed by metabolic disorders, and early marked cerebral atrophy. Metabolic disorders were corrected after feeding with extensively hydrolyzed protein formula. Seizures started at the 19th day of life. Interictal VEEG showed a suppression-burst (SB) pattern and ictal VEEG revealed myoclonic seizures that were compatible with early myoclonic encephalopathy (EME). She had frequent myoclonic seizures resistant to multi-antiepileptic drugs including phenobarbital, levetiracetam and oxcarbazepine, and soon developed into convulsive status epilepticus. At 7 months of age, she had severe developmental delay, and developed infantile spasms. Our case report expands the phenotypic spectrum of the PCH6, meanwhile, RARS2 should be considered be a causative gene in patients with EME.
\end{abstract}

Keywords: Mitochondrial arginyl-tRNA synthetase 2 (RARS2); early myoclonic encephalopathy (EME); case report

Submitted Mar 25, 2020. Accepted for publication Jul 31, 2020.

doi: $10.21037 / \mathrm{tp}-20-110$

View this article at: http://dx.doi.org/10.21037/tp-20-110

\section{Introduction}

The mitochondrial arginyl - tRNA synthetase 2 (RARS2) gene encodes mitochondrial arginine-tRNA synthetase. Patients with RARS2 gene variants have been classically described in pontocerebellar hypoplasia type 6 (PCH6) that is characterized by early onset seizures, progressive microcephaly, and developmental delay.

EME is a rare early-onset epileptic syndrome, which characterized by frequent intractable seizures and severe developmental impairment. Thus far, EME caused by RARS2 variants has not yet been reported. Here we described a female term neonate with heterozygous variants of the RARS2 gene who was presented with EME. We present the following case in accordance with the CARE reporting checklist (available at http://dx.doi.org/10.21037/ tp-20-110).

\section{Case presentation}

A female term neonate was the first pregnancy for a nonconsanguineous couple. She was delivered at 39 weeks of gestation via cesarean section for intrauterine fetal 


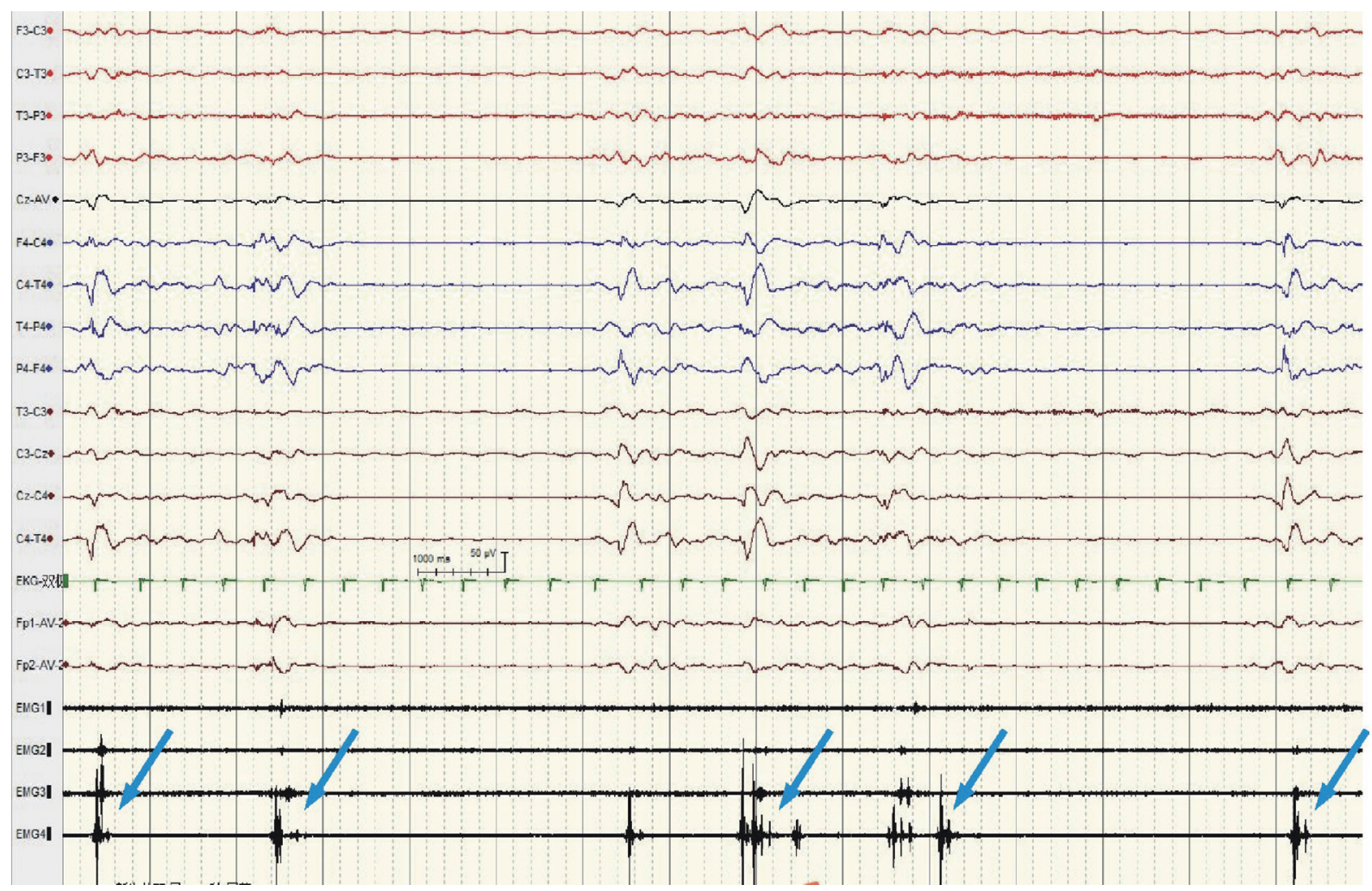

Figure 1 VEEG at DOL24. International 10-20 system modified for neonates, HHF: $70 \mathrm{~Hz}$, Sensitivity $10 \mu \mathrm{V} / \mathrm{mm}$. Interictal EEG showed a symmetric and synchronous SB pattern. Ictal EEG revealed frequent myoclonic seizures often coincided the burst phases. Arrow: myoclonic seizures. Electromyogram 1 (EMG1)/EMG2: distal left/right upper extremity muscles. EMG3/EMG4: distal left /right lower extremity muscles.

distress and premature rupture of fetal membranes with weight of $3,300 \mathrm{~g}(50 \%)$, length of $50 \mathrm{~cm}(50 \%)$ and head circumference of $34 \mathrm{~cm}(50 \%)$ at birth. Her Apgar scores were $8,10,10$ respectively. Her parents were healthy and denied any family history of genetic disease. Her mother had regular prenatal examinations, and denied any abnormal results, and denied the history of exposure to any harmful substances.

At day of life 2 (DOL2), groan was manifested, which initially was attributed to respiratory infection. After breast and artificial feeding, hypoglycemia, metabolic acidosis and hyperammonemia were noted repetitively at DOL7 and improved considerably after fasting and parenteral nutrition. Concentrations of plasma ammonia and blood lactic acid remained normal value when she was given extensively hydrolyzed protein formula.

At DOL19, she manifested partial or fragmentary erratic myoclonus, sometimes massive myoclonus, and muscular hypertonia. Blood tandem mass spectrometry and urine gas chromatography-mass spectrometry were normal. Cerebrospinal fluid (CSF) analyses were unremarkable.

Interictal video-electroencephalogram (VEEG) at DOL24 showed a symmetric and synchronous suppressionburst (SB) pattern during all wakefulness and sleep, which consisted of bursts lasting 2 to 6 seconds followed by almost isoelectric periods lasting 3 to 96 seconds. Ictal VEEG revealed frequent myoclonic seizures often coincided the burst phases (Figure 1).

Brain magnetic resonance imaging (MRI) at DOL27 demonstrated enlarged ventricular system, widened extracerebral space at the frontal, temporal and sylvian fissure but more obviously at left side, significantly small vessels in bilateral temporal region and bilateral symmetric hypomyelination in the posterior limb of internal capsule (Figure 2).

Multi-antiepileptic drugs therapy including phenobarbital 

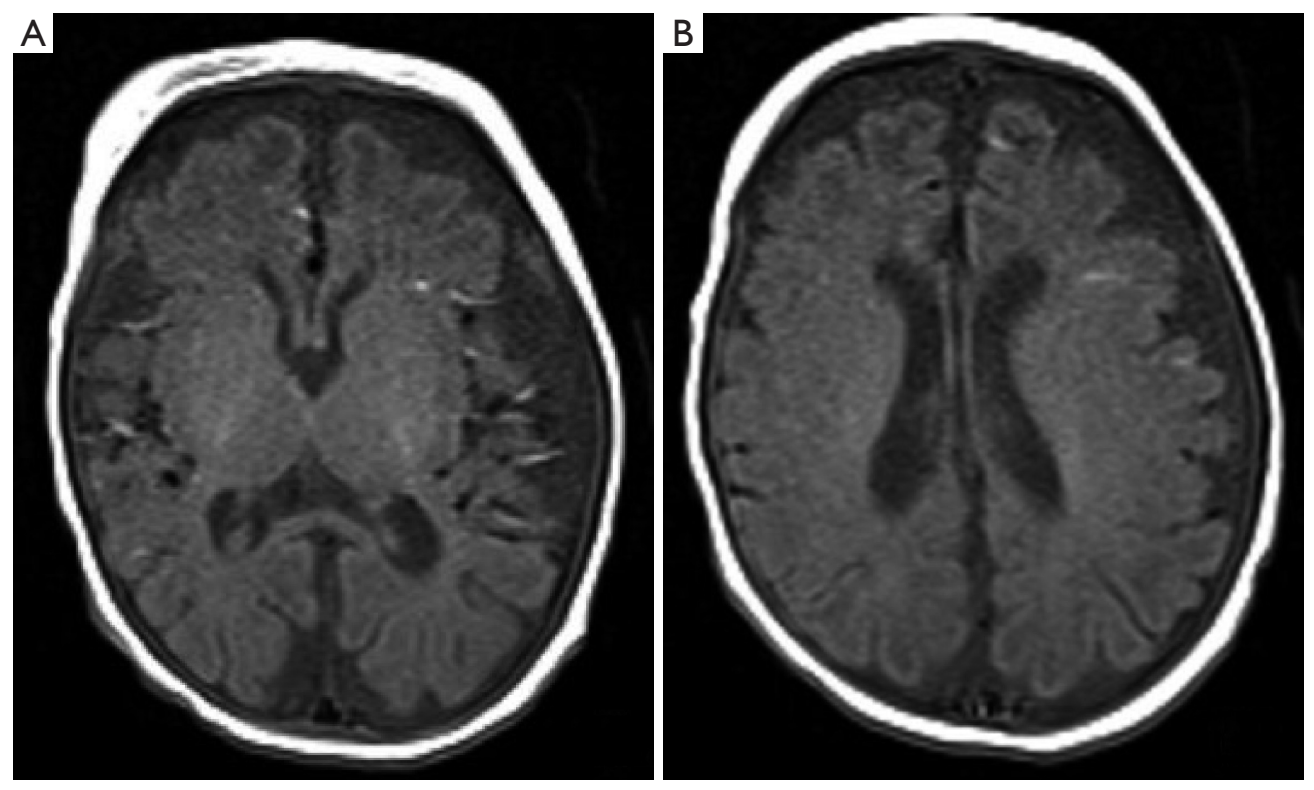

Figure 2 Brain MRI at DOL27 (axial T1-weighted imaging). (A,B) Marked supratentorial atrophy, obviously at the left side; significant small vessels in bilateral temporal region and hypomyelination in the posterior limb of internal capsule.

( $5 \mathrm{mg} / \mathrm{kg} / \mathrm{d}$, used at DOL21, stopped at 5 months of age), levetiracetam $(30 \mathrm{mg} / \mathrm{kg} / \mathrm{d}$, added at DOL 29, stopped at 5 months of age $)$ and oxcarbazepine $(20 \mathrm{mg} / \mathrm{kg} / \mathrm{d}$, added at DOL 38, stopped at 5 months of age) were not effective. She soon developed into convulsive status epilepticus. Trio-whole-exome sequencing revealed compound heterozygous variants in $R A R S 2$, she carried a pathogenic missense mutation [NM_020320: exon10: c.773G $>$ A (p. R258H) Maternal] (1) in combination with a novel frameshift mutation (NM_020320:exon4:c.282_285delAG AG Paternal), which was confirmed by Sanger sequencing (Figure 3).

Her parents discharged automatically with antiepileptic drugs including phenobarbital, levetiracetam and oxcarbazepine when she was 2 months of age. Followup revealed that any antiepileptic drugs was withdrawn at 5 months of age because ineffective. She developed infantile spasm at 7 months of age, and had a severe developmental delay (at 7 months of age, she had poor visual fixation without following, had poor head control, was not sitting, grasping and turning over) (Figure 4).

All procedures performed in studies involving human participants were in accordance with the ethical standards of the institutional and/or national research committee(s) and with the Helsinki Declaration (as revised in 2013). Written informed consent was obtained from the patient.

\section{Discussion}

The RARS2 gene encodes mitochondrial arginine-tRNA synthetase, which was identified by Edvardson et al. in 2007 (2). Variants in RARS2 gene are associated with pontocerebellar hypoplasia type 6 (PCH6; MIM 611523), a rare mitochondrial encephalopathy with phenotype characterized by early onset seizures, profound developmental delay, and progressive pontocerebellar atrophy.

Our patient manifests a series of clinical features common to previously reported (1-6), including early onset of disease, neonatal hypoglycemia, early raised serum and/or CSF lactate and pharmaco-resistant epileptic encephalopathy.

Groan after birth was the first symptom manifested in our patient, while respiratory symptom was seldom described previously. Edvardson et al. (2) have reported one patient had a progression from a single apneic episode at DOL2 to respiratory failure at the age of 3 weeks. The probable causes were supposed to be metabolic acidosis, insufficient mitochondrial energy production or central nervous dysfunction.

From the characteristic features of RARS2-PCH6 on neuroimaging perspective, MR imaging of most patients within the first months of life revealed cerebral and/or 
A

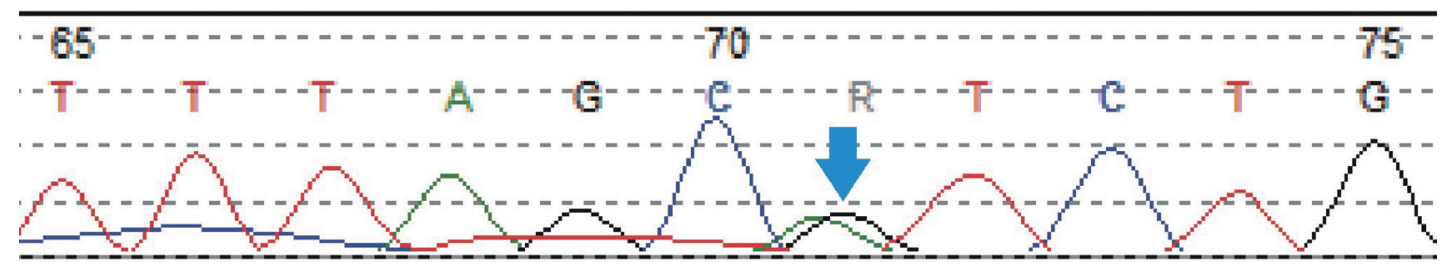

B

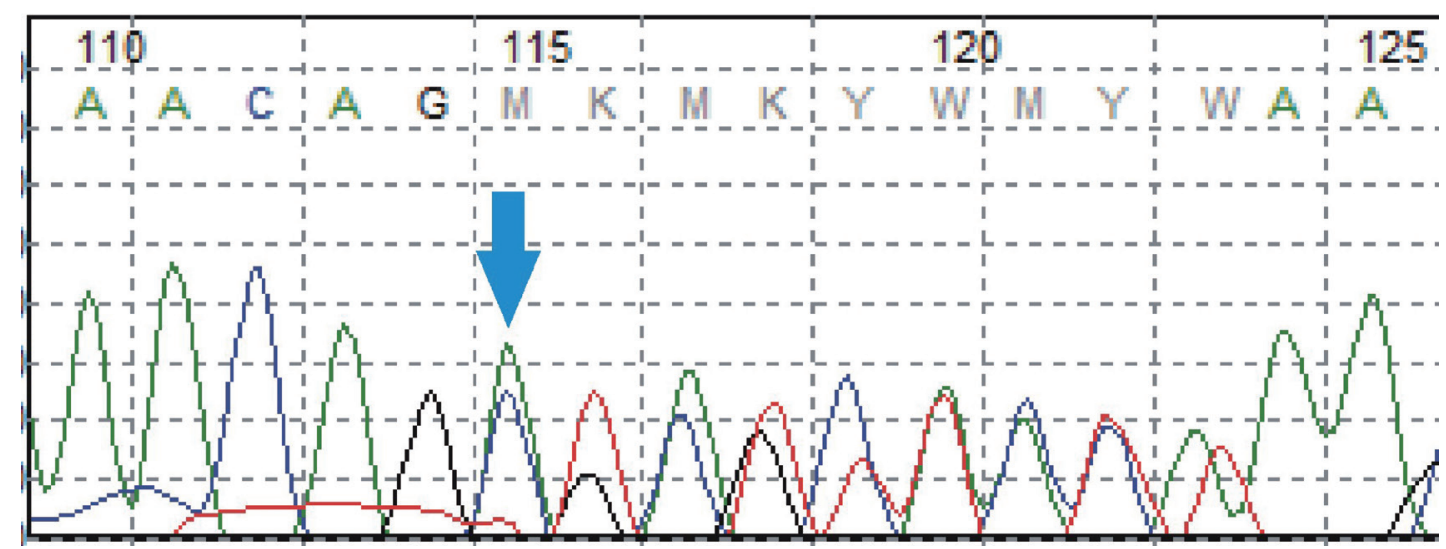

Figure 3 Image obtained on Sanger sequencing. A depicts a $(\mathrm{G}>\mathrm{A})$ variant in the $R A R S 2$ gene from the mother; B depicts a (c.282_285delAGAG) variant in the RARS2 gene from the father.

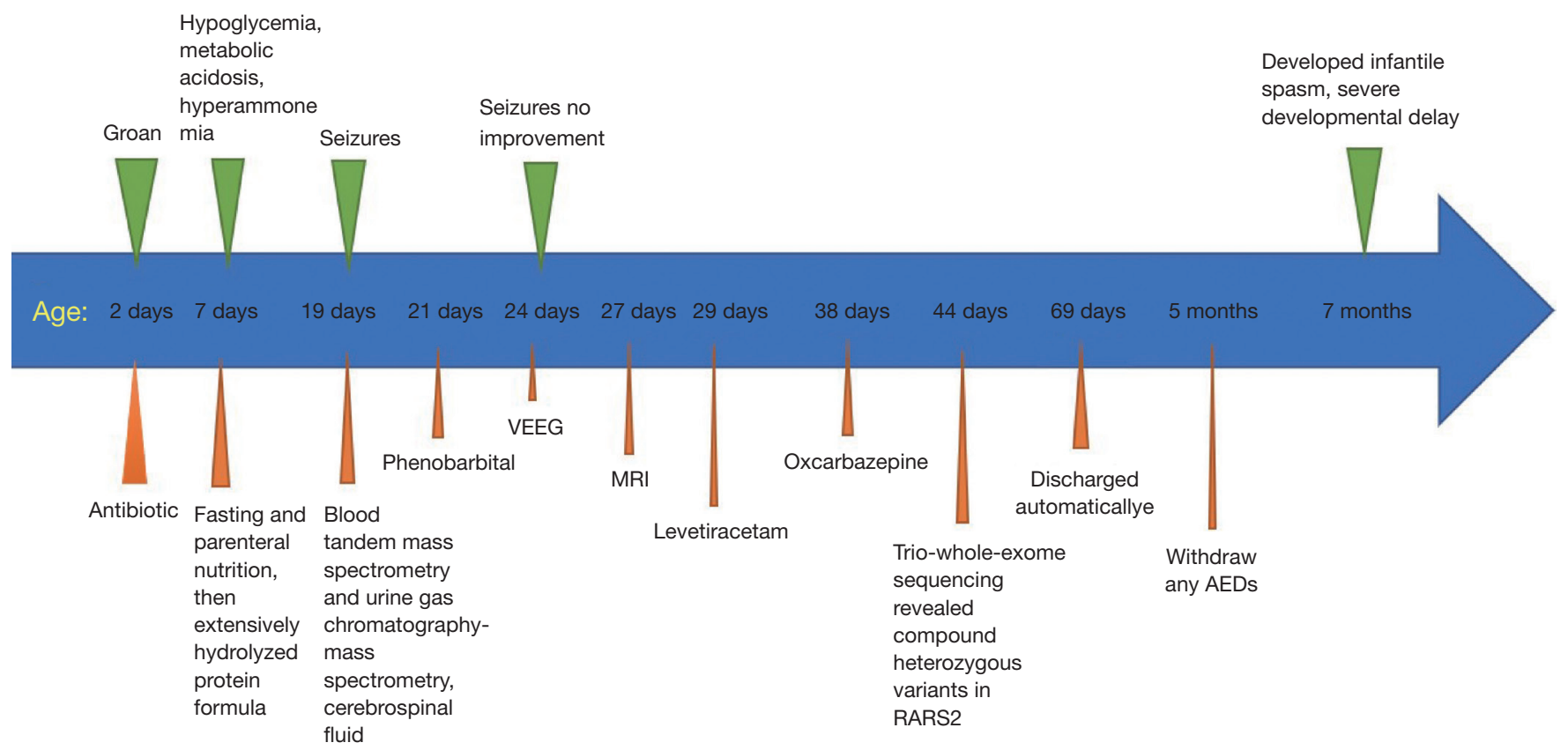

Figure 4 Timeline of this case. AEDs, antiepileptic drugs; MRI, magnetic resonance imaging; VEEG, video-electroencephalogram. 
pontocerebellar atrophy that may be progressive over time (2-6). Other imaging features described in reported patients include subdural effusions and abnormal myelination $(1,6)$. To the best of our knowledge, significant small vessels revealed in bilateral temporal region by MRI have not been reported so far. This phenomenon is unclear and we speculate that the blood vessels may have not decreased accordingly with cerebral atrophy.

The electroclinical spectrum of Patients with RARS2$\mathrm{PCH}$ manifest multiple different seizure types including generalized tonic-clonic, focal, and myoclonic seizures. Our patient presented with EME, which has not been reported in RARS2-PCH6 previously. EME is a rare early-onset epileptic syndrome that usually begins in neonatal period. It is characterized by frequent myoclonus and partial seizures and by an SB pattern in the EEG (7).

Although underlying pathologies of EME are not demonstrated in the majority of cases, the most striking feature of the high incidence of EME in familial cases with metabolic disorders suggests that inborn errors of metabolism and/or genetic factors play a prominent role (7). Metabolic abnormalities such as nonketotic hyperglycinemia and disorders of amino acid metabolism were frequently described in the cases with EME (8-10). Other related genes, including CDKL5 (11), KCNQ2 (12), ErbB4 (13), SCN1A (14), GABRB2 (15), SIK1 (16), AMT (17) and $\operatorname{SLC} 25 A 22(18)$, as well as a $9 \mathrm{q} 33$ - $\mathrm{q} 34$ deletion including STXBP1 and SPTAN1 (19), have been reported to be associated with EME.

Mathew et al. (20) has reported that two siblings with $R A R S 2$ variants started myoclonic jerks after birth, however, related MRI and EEG features during the period of neonate were not provided in their report. Nuclear mitochondrial genes, such as $A M T$ encodes an enzyme system for cleavage of glycine which is confined to the mitochondria and SLC25A22 encodes mitochondrial carriers that transport a variety of metabolites across the inner mitochondrial membrane. RARS2 encodes the mitochondrial aminoacyl tRNA synthetase enzyme and play an important role in mRNA translation, a key process in maintaining cell integrity, so there may be some correlation in the pathogenesis of EME caused by nuclear mitochondrial gene variants.

In our case, we diagnosed EME by VEEG and known she had a poor outcome with refractory epilepsy and severe developmental impairment. The main causes of EME were metabolic factors and genetic factors (7). She had no obvious abnormal metabolism. Genetic testing helped us to find the cause in such a complex congenital disease. However, there are no effective treatments at present. The parents also refused to accept any other treatments, such as adrenocorticotropic hormone, ketogenic diet, and vagus nerve stimulation. So we couldn't obtain the data of following VEEG, MRI and general clinical characteristics.

\section{Conclusions}

This case report expands the phenotypic spectrum of the $R A R S 2$ variants. Meanwhile, RARS2 should be considered be a causative gene in patients with EME.

\section{Acknowledgments}

The authors are thankful for the doctors and nurses of the Department of Neonatology, Children's Hospital of Fudan University, Shanghai, China.

Funding: None.

\section{Footnote}

Reporting Checklist: The authors have completed the CARE reporting checklist. Available at http://dx.doi.org/10.21037/ tp-20-110

Conflicts of Interest: All authors have completed the ICMJE uniform disclosure form (available at http://dx.doi. org/10.21037/tp-20-110). The authors have no conflicts of interest to declare.

Ethical Statement: The authors are accountable for all aspects of the work in ensuring that questions related to the accuracy or integrity of any part of the work are appropriately investigated and resolved. All procedures performed in studies involving human participants were in accordance with the ethical standards of the institutional and/or national research committee(s) and with the Helsinki Declaration (as revised in 2013). Written informed consent was obtained from the patient. A copy of the written consent is available for review by the Editors-in-Chief of this journal.

Open Access Statement: This is an Open Access article distributed in accordance with the Creative Commons Attribution-NonCommercial-NoDerivs 4.0 International License (CC BY-NC-ND 4.0), which permits the noncommercial replication and distribution of the article with 
the strict proviso that no changes or edits are made and the original work is properly cited (including links to both the formal publication through the relevant DOI and the license). See: https://creativecommons.org/licenses/by-nc-nd/4.0/.

\section{References}

1. Kastrissianakis K, Anand G, Quaghebeur G, et al. Subdural effusions and lack of early pontocerebellar hypoplasia in siblings with RARS mutations. Arch Dis Child 2013;98:1004-7.

2. Edvardson S, Shaag A, Kolesnikova O, et al. Deleterious mutation in the mitochondrial arginyl-transfer RNA synthetase gene is associated with pontocerebellar hypoplasia. Am J Hum Genet 2007;81:857-62.

3. Cassandrini D, Cilio MR, Bianchi M, et al. Pontocerebellar hypoplasia type 6 caused by mutations in RARS2: Definition of the clinical spectrum and molecular findings in five patients. J Inherit Metab Dis 2013;36:43-53.

4. Li Z, Schonberg R, Guidugli L, et al. A novel mutation in the promoter of RARS2 causes pontocerebellar hypoplasia in two siblings. J Hum Genet 2015;60:363-9.

5. Ngoh A, Bras J, Guerreiro R, et al. RARS2 mutations in a sibship with infantile spasms. Epilepsia 2016;57:e97-102.

6. Zhang J, Zhang Z, Zhang Y, et al. Distinct magnetic resonance imaging features in a patient with novel RARS2 mutations: A case report and review of the literature. Exp Ther Med 2018;15:1099-104.

7. Ohtahara S, Yamatogi Y. Epileptic encephalopathies in early infancy with suppression-burst. J Clin Neurophysiol 2003;20:398-407.

8. Lombroso CT. Early myoclonic encephalopathy, early infantile epileptic encephalopathy, and benign and severe infantile myoclonic epilepsies: a critical review and personal contributions. J Clin Neurophysiol 1990;7:380-408.

9. Lee WT. Disorders of amino acid metabolism associated with epilepsy. Brain Dev 2011;33:745-52.

10. Aukett A, Bennett MJ, Hosking GP. Molybdenum co-

Cite this article as: $\mathrm{Xu} \mathrm{Y,} \mathrm{Wu} \mathrm{BB,} \mathrm{Wang} \mathrm{HJ,} \mathrm{Zhou} \mathrm{SZ,}$ Cheng GQ, Zhou YF. A term neonate with early myoclonic encephalopathy caused by RARS2 gene variants: a case report. Transl Pediatr 2020;9(5):707-712. doi: 10.21037/tp-20-110 factor deficiency: an easily missed inborn error of metabolism. Dev Med Child Neurol 1988;30:531-5.

11. Takeda K, Miyamoto Y, Yamamoto H, et al. Clinical features of early myoclonic encephalopathy caused by a CDKL5 mutation. Brain Dev 2020;42:73-6.

12. Kojima K, Shirai K, Kobayashi M, et al. A patient with early myoclonic encephalopathy (EME) with a de novo KCNQ2 mutation. Brain Dev 2018;40:69-73.

13. Backx L, Ceulemans B, Vermeesch JR, et al. Early myoclonic encephalopathy caused by a disruption of the neuregulin-1 receptor ErbB4. Eur J Hum Genet 2009; 17:378-82.

14. Ishikawa $\mathrm{N}$, Tateishi $\mathrm{Y}$, Tani $\mathrm{H}$, et al. Successful treatment of intractable life-threatening seizures with perampanel in the first case of early myoclonic encephalopathy with a novel de novo SCN1A mutation. Seizure 2019;71:20-3.

15. Ishii A, Kang JQ, Schornak CC, et al. A de novo missense mutation of GABRB2 causes early myoclonic encephalopathy. J Med Genet 2017;54:202-11.

16. Hansen J, Snow C, Tuttle E, et al. De novo mutations in SIK1 cause a spectrum of developmental epilepsies. Am J Hum Genet 2015;96:682-90.

17. Belcastro V, Barbarini M, Barca S, et al. A novel AMT gene mutation in a newborn with nonketotic hyperglycinemia and early myoclonic encephalopathy. Eur J Paediatr Neurol 2016;20:192-5.

18. Cohen R, Basel-Vanagaite L, Goldberg-Stern H, et al. Two siblings with early infantile myoclonic encephalopathy due to mutation in the gene encoding mitochondrial glutamate/H+ symporter SLC25A22. Eur J Paediatr Neurol 2014;18:801-5.

19. Aravindhan A, Shah K, Pak J, et al. Early-onset epileptic encephalopathy with myoclonic seizures related to 9q33.3-q34.11 deletion involving STXBP1 and SPTAN1 genes. Epileptic Disord 2018;20:214-8.

20. Mathew T, Avati A, D'Souza D, et al. Expanding spectrum of RARS2 gene disorders: Myoclonic epilepsy, mental retardation, spasticity, and extrapyramidal features. Epilepsia Open 2018;3:270-5. 